\title{
Volumetric Blood Flow in Transjugular Intrahepatic Portosystemic Shunt Revision Using 3-Dimensional Doppler Sonography
}

\author{
Stephen Z. Pinter, PhD, Jonathan M. Rubin, MD, PhD, Oliver D. Kripfgans, PhD, Paula M. Novelli, MD, \\ Mario Vargas-Vila, PhD, Anne L. Hall, PhD, J. Brian Fowlkes, PhD
}

Received December 16, 2013, from the Department of Radiology, University of Michigan, Ann Arbor, Michigan USA (S.Z.P., J.M.R., O.D.K., P.M.N., M.V.-V., J.B.F.); and GE Healthcare, Milwaukee, Wisconsin USA (A.L.H.). Revision requested February 16, 2014. Revised manuscript accepted for publication May 21, 2014.

This research was funded in part by the Michigan Institute for Clinical and Health Research (National Institutes of Health grant UL1RR024986), the University of Michigan Health System (radiology seed grant), and a research grant from GE Healthcare.

Address correspondence to Oliver D. Kripfgans, PhD, Department of Radiology, University of Michigan, 3232 Medical Sciences Building I, 1301 Catherine St, Ann Arbor, MI 48109-5667 USA.

E-mail: oliver.kripfgans@umich.edu

\section{Abbreviations}

4D, 4-dimensional; 3D, 3-dimensional; TIPS, transjugular intrahepatic portosystemic shunt

doi:10.7863/ultra.34.2.257
Objectives-Three-dimensional (3D)/4-dimensional (4D) sonographic measurement of blood volume flow in transjugular intrahepatic portosystemic shunt revision with the intention of objective assessment of shunt patency.

Methods - A total of 17 patients were recruited ( 12 male and 5 female; mean age, 55 years; range, 30-69 years). An ultrasound system equipped with a 2.0-5.0-MHz probe was used to acquire multivolume $3 \mathrm{D} / 4 \mathrm{D}$ color Doppler data sets to assess prerevision and postrevision shunt volume flow. Volume flow was computed offline based on the principle of surface integration of Doppler-measured velocity vectors in a lateral-elevational c-surface positioned at the color flow focal depth (range, $8.0-11.5 \mathrm{~cm}$ ). Volume flow was compared to routine measurements of the prerevision and postrevision portosystemic pressure gradient. Prerevision volume flow was compared with the outcome to determine whether a flow threshold for revision could be defined.

Results - Linear regression of data from revised transjugular intrahepatic portosystemic shunt cases showed an inverse correlation between the mean-normalized change in prerevision and postrevision shunt volume flow and the mean-normalized change in the prerevision and postrevision portosystemic pressure gradient $\left(r^{2}=0.51 ; P=.020\right)$. Increased shunt blood flow corresponded to a decreased pressure gradient. Comparison of prerevision flows showed preliminary threshold development at $1534 \mathrm{~mL} / \mathrm{min}$, below which a shunt revision may be recommended $(P=.21$; area under the receiver operating characteristic curve $=0.78$ ).

Conclusions - Shunt volume flow measurement with 3D/4D Doppler sonography provides a potential alternative to standard pulsed wave Doppler metrics as an indicator of shunt function and predictor of revision.

Key Words - color flow; Doppler sonography; portal hypertension; power Doppler sonography; transjugular intrahepatic portosystemic shunt revision; vascular ultrasound; volume flow

$\mathrm{T}$ ransjugular intrahepatic portosystemic shunts (TIPSs) are used in the management of portal hypertension and related portal hypertensive complications such as variceal hemorrhage and ascites. ${ }^{1-6}$ Thrombosis or stenosis of the shunt is a principal concern, as demonstrated by primary shunt patency rates: $25 \%$ to $66 \%, 5 \%$ to $42 \%, 21 \%, 13 \%$, and $13 \%$ at years 1 through 5 , respectively. ${ }^{3,4,7-13}$ Primary shunt patency rates have been updated by the introduction of covered stents, which show lower rates of 
failure than uncovered stents. A recent study by Bureau et $\mathrm{al}^{14}$ compared outcomes in 39 patients with covered stents and 41 patients with uncovered stents. Covered-stent shunt dysfunction was shown in $13 \%$ of patients based on the criteria of greater than $50 \%$ stenosis or a portosystemic pressure gradient of greater than $12 \mathrm{~mm} \mathrm{Hg}$ after a median follow-up of 300 days. Other investigators have shown covered stent dysfunction rates between $8 \%$ and $20 \%$ at 1 year. ${ }^{15-19}$ However, approximately $20 \%$ of TIPS procedures in the United States still use uncovered stents. ${ }^{20}$

Interventional revision prolongs patency and if performed before complete occlusion yields increased primaryassisted patency rates: $80 \%$ to $85 \%, 61 \%$ to $79 \%, 46 \%$ to $87 \%, 42 \%$, and $36 \%$ at years 1 through 5 , respectively. $4-711,13$ Therefore, shunt dysfunction must be identified promptly and managed appropriately to define a therapeutic window for revision.

Transjugular intrahepatic portosystemic shunt screening identifies cases that require revision, but current sonographic assessment techniques have variable sensitivity and specificity, and venography, the reference standard for detecting shunt dysfunction, is invasive and unsuitable for routine use. ${ }^{4,6}$ Doppler sonography is the primary imaging modality used for TIPS patency screening. ${ }^{4,6}$ Although several flow velocity criteria for defining TIPS failure have been proposed in the literature, each has proponents and detractors, and a nonuniform association exists between flow velocity and shunt patency. ${ }^{4,6,21-27}$ Literature has also demonstrated a poor correlation $(r=-0.11)$ between flow velocity and the portosystemic pressure gradient. ${ }^{28}$ Variability in the flow velocity estimate is motivated by several objective and subjective factors: resistance of the overall flow path, Doppler angle, cylindrically asymmetric flow profile, internal aliasing, and shunt morphologic features such as overlapping stents and curves in the shunt. Individually or collectively, these factors can yield velocity fluctuation along the length of the shunt.

Volume flow is an alternative metric to flow velocity that may be used to assess shunt patency. ${ }^{29-31}$ In a TIPS, since there is only one inflow and one outflow, volume flow is conserved and thus equivalent everywhere along the path of the shunt. Therefore, volume flow provides a potentially robust metric for evaluating shunt patency.

The proposed 3-dimensional (3D)/4-dimensional (4D) volume flow measurement technique-based on surface integration of Doppler-measured velocity vectorsis noninvasive, objective, and independent of all traditional pulsed wave Doppler assumptions. ${ }^{32-37}$ Measurement of TIPS volume flow is demonstrated in patients undergoing revision and compared to standard measurements of the revision portosystemic pressure gradient. Volume flow measurements are also evaluated on the basis of prerevision shunt flow and outcome to determine whether a flow threshold for revision could be defined. The hypothesis of this study was that the proposed 3D/4D sonographic volume flow measurement technique provides straightforward, objective, and reliable assessment of shunt flow as a means for determining patency and defining a therapeutic strategy for revision.

\section{Materials and Methods}

\section{Clinical and Experimental Procedures}

A total of 17 patients admitted for TIPS revision were consecutively enrolled from the University of Michigan Medical Center's interventional radiology service in this prospective study. This patient population was selected because prerevision and postrevision portosystemic pressure gradient measurements would be available as a reference standard for comparison with the volume flow estimates. Patient demographics and clinical characteristics are summarized in Tables 1 and 2. Table 2 shows a total prospective patient count of 19 because patient 3 was scanned 3 times on separate hospital visits (3A, 3B, and 3C). Patients included in the study were referred to interventional radiology with clinical signs of shunt dysfunction (reaccumulation of ascites and hepatic hydrothorax) or sonographic findings showing a temporal change in shunt velocities or flow patterns. Each patient provided fully informed written consent to an Institutional Review Board-approved protocol involving transcutaneous measurement of blood volume flow using sonography. Patients unwilling to sign consent were excluded from the study.

Table 1. Patient Demographics

\begin{tabular}{lc}
\hline Characteristic & Value \\
\hline Male/female, $\mathrm{n}$ & $12 / 5$ \\
Mean age (range), $\mathrm{y}$ & $55(30-69)$ \\
Etiology of cirrhosis, $\mathrm{n}$ & \\
Alcohol & 4 \\
Hepatitis C & 3 \\
Sclerosing cholangitis & 1 \\
Nonalcoholic steatohepatitis/cryptogenic & 6 \\
Budd-Chiari & 1 \\
Mesenteric thrombosis & 1 \\
Congenital hepatic fibrosis & 1 \\
Indication, $\mathrm{n}$ & \\
Varices & 6 \\
Ascites & 10 \\
Both & 1 \\
\hline
\end{tabular}


From the 17-patient population, a total of 20 TIPS cases were obtained because patient 5 had 2 TIPSs and patient 3 was scanned 3 times on separate hospital visits. The 20 cases can be categorized as follows: 12 TIPSs revised, 3 TIPSs not revised, and 5 TIPS cases omitted. Cases were omitted for one of the following reasons, none of which was due to the surface integration of Doppler-measured velocity vector volume flow technique, but to unrelated factors: (1) data acquisition error, (2) inadequate color flow image quality, (3) high body mass index (ie, poor acoustic access), and (4) fully thrombosed shunt (ie, lack of a prerevision pressure gradient). These cases are marked with an asterisk in Table 2 . In addition, 2 revised cases (patients 5 and 11) were excluded, as described in "Results."

Shunt volume flow was assessed twice for each patient with one flow measurement immediately before revision $(<2$ hours) and one flow measurement immediately subsequent to revision ( $<2$ hours). All patients received nothing by mouth for at least 6 hours before prerevision volume flow measurement and maintained receiving nothing by mouth for the postrevision scan.

Transjugular intrahepatic portosystemic shunt revision followed the standard interventional radiology protocol at the University of Michigan Medical Center (P.M.N., with 11 years of experience). The decision for angioplasty was based on venographically visible stenosis. Stents were dilated with a 4-cm-long balloon equal in diameter to the original stent. Viatorr stents (Gore Medical, Flagstaff, AZ) were dilated to $10 \mathrm{~mm}$. Angioplasty was followed by stent revision if venographic success was not achieved. A peak systolic gradient between the right portal vein and right atrium of greater than $12 \mathrm{~mm} \mathrm{Hg}$ defined TIPS dysfunction. Venography was performed in all patients to document the stent luminal caliber, flow reversal in the portal circulation, and antegrade flow in hepatic portal venous branches. Pressure measurements were made under quiet tidal respiration and averaged over 20 seconds.

A LOGIQ 9 ultrasound system (GE Healthcare, Milwaukee, WI) equipped with a $2.0-5.0-\mathrm{MHz}$ probe (4D3CL) was used to acquire multivolume 4D (near realtime 3D) color Doppler data sets. Each multivolume data set consisted of 30 image volumes acquired sequentially at a rate of 1 volume every 10 seconds. This sequence corresponded to an acquisition rate of $0.1 \mathrm{~Hz}$, although the acquisitions were not continuous. Patients held their breath during a volume acquisition and breathed between acquisitions. There was also a time delay for the $3 \mathrm{D}$ reconstruction between acquisitions. Since TIPS flow can be respiratory phase dependent, ${ }^{38}$ patients were instructed to simply stop breathing with no bias toward inspiration or expiration. Multi-

Table 2. Patient Clinical Characteristics

\begin{tabular}{|c|c|c|c|c|c|c|c|}
\hline Patient & $\begin{array}{c}\text { Time After } \\
\text { TIPS } \\
\text { Creation, mo }\end{array}$ & $\begin{array}{c}\text { Time After } \\
\text { Most Recent } \\
\text { Revision, mo }\end{array}$ & $\begin{array}{l}\text { Stent } \\
\text { Type }\end{array}$ & $\begin{array}{c}\text { Clinical } \\
\text { Indication } \\
\text { for Revision }\end{array}$ & $\begin{array}{c}\text { Morphologic } \\
\text { Indication } \\
\text { for Revision }\end{array}$ & $\begin{array}{c}\text { Abnormal } \\
\text { Sonographic } \\
\text { Findings }^{a}\end{array}$ & Revision Type \\
\hline $1^{*}$ & 68 & 62 & VSG & Asymptomatic & None & Yes & None \\
\hline $2^{\star}$ & 1 & & VSG & Refractory ascites & None & Yes & None \\
\hline $3(3 \mathrm{~A})$ & 17 & 6 & VSG & Refractory ascites & Stenosis & Yes & Balloon angioplasty \\
\hline 4 & 143 & & BMS & Asymptomatic & Stenosis & Yes & Balloon angioplasty \\
\hline 5 & 60 & & VSG & Refractory ascites & Occlusion & Yes & Balloon angioplasty \\
\hline $6^{\star}$ & 27 & & VSG & Refractory ascites & None & Yes & None \\
\hline $7^{\star}$ & 1 & & BMS & Refractory ascites & Occlusion & Yes & Stent graft implantation \\
\hline $8^{*}$ & 20 & 6 & VSG & Refractory ascites & Occlusion & Yes & Bare stent implantation \\
\hline 9 & 24 & & VSG & Refractory ascites & Stenosis & No & Balloon angioplasty \\
\hline 10 & 24 & & VSG & Refractory ascites & Stenosis & Yes & Bare stent implantation \\
\hline 11 & 112 & 104 & BMS & Refractory ascites & Stenosis & Yes & Balloon angioplasty \\
\hline 12 & 12 & & VSG & Refractory ascites & Stenosis & Yes & Bare stent implantation \\
\hline 14 & 42 & 14 & VSG & Refractory ascites & Stenosis & Yes & Balloon angioplasty \\
\hline $15(3 B)$ & 23 & 5 & VSG & Refractory ascites & Stenosis & Yes & Balloon angioplasty \\
\hline 16 & 166 & & VSG & Asymptomatic & None & Yes & Balloon angioplasty \\
\hline 17 & 12 & & VSG & Refractory ascites & None & No & Balloon angioplasty \\
\hline 18 & 12 & & VSG & Asymptomatic & None & Yes & None \\
\hline $19(3 C)$ & 28 & 5 & VSG & Refractory ascites & Stenosis & Yes & Bare stent \\
\hline
\end{tabular}

BMS indicates bare metal stent; 3A, 3B, and 3C, multiple studies in patient 3; and VSG, Viatorr stent-graft.

${ }^{a}$ Abnormal sonographic findings consisted of 1 or more of the following criteria: (1) changes in baseline velocities, (2) reversal of flow in the left or right portal vein away from the TIPS, and (3) absence of flow in the TIPS.

*Omitted for reasons described in "Materials and Methods." 
volume data sets were processed offline by exporting LOGIQ 9 4D duplex mode raw DICOM data (Digital Imaging and Communications in Medicine) (Medical Imaging \& Technology Alliance, Arlington, VA) and the associated scanner acquisition settings for prospective analysis.

Figure 1 illustrates the imaging geometry required for the proposed volume flow technique. The position and tilt angle of the probe must be adjusted to visualize the shunt cross section in the lateral-elevational imaging surface. Although the shunt is required to intersect the c-surface in cross section (Figure 1A), the volume flow method is independent of the angle at which the shunt intersects the c-surface. For example, the shunt cross section can vary from circular to ellipsoidal (Figure 1B), and each geometry will yield an identical volume flow estimate. The c-surface was typically positioned near the middle of the stent and as close as possible to the color flow focus, which ranged in depth from 8.0 to $11.5 \mathrm{~cm}$ ( $14.5 \mathrm{~cm}$ in 1 case) depending on the position of the shunt, patient's body mass index, and presence of overlying ascites. For the 8.0 - to $11.5-\mathrm{cm}$ depth range, the c-surface consisted of a lateral pixel size range of 1.2 to 1.5 $\mathrm{mm}$ and an elevational pixel size range of 1.5 to $2.0 \mathrm{~mm}$.

Volume flow for each multivolume data set was computed offline (S.Z.P.) using custom algorithms written in MATLAB (The MathWorks, Natick, MA), which implemented the principle of surface integration of Dopplermeasured velocity vectors. ${ }^{32,34}$ The computation c-surface was defined at, or near, the color flow focus of the acquired
$3 \mathrm{D}$ volume. Power Doppler data were used as a correction factor to compensate for partial volume effects by weighting flow estimates in reference to pixels containing 100\% blood. ${ }^{33,35,36}$ Principles of the volume flow computation method are described later in this section.

For each 30-volume data set, volume flow was computed on a volume-by-volume basis to yield 30 individual estimates that were subsequently averaged to produce the overall flow in the shunt. Volume-by-volume flow computation is advantageous because it allows for shunt motion (respiration, bulk motion, and probe movement) within the scanning volume of interest between acquisitions. In some data sets, however, a few individual volumes were discarded due to movement during an acquisition.

Two segmentation methods were used to exclude unrelated vasculature and local color flow artifacts from the c-surface. First, directional criteria were used to automatically differentiate shunt flow from any adjacent arterial flow. Second, manual segmentation (S.Z.P.) placed a broad outline around the shunt and excluded extraneous vessels and artifacts in the volume of interest.

Prerevision and postrevision shunt volume flow measurements made by sonography were compared to routine prerevision and postrevision portosystemic pressure gradients measured via a catheter using an electronic manometer. The volume flow metric was defined as the mean-normalized change in prerevision and postrevision shunt volume flow $\left(\Delta Q / Q_{\text {avg }}\right)$, and the portosystemic pressure gradient metric was defined as the mean-normalized

Figure 1. A, Imaging geometry required for the proposed volume flow measurement technique. The probe is oriented such that the TIPS intersects the c-surface (lateral-elevational surface) in cross section. B. The angle of c-surface intersection is an independent variable. The TIPS can intersect the c-surface at angles that yield circular $\left(\theta_{1}\right)$ to ellipsoidal $\left(\theta_{2}\right.$ and $\left.\theta_{3}\right)$ geometries, and all cases would yield an identical volume flow estimate.

A

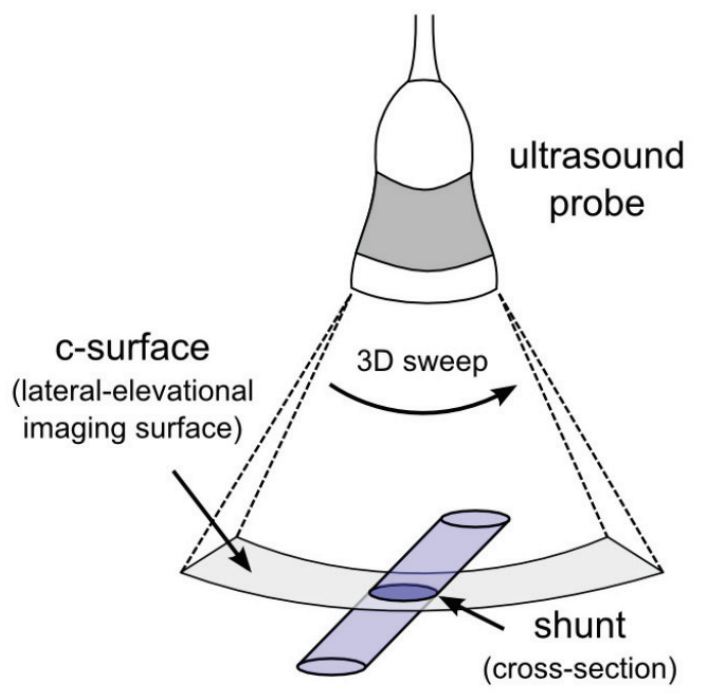

B

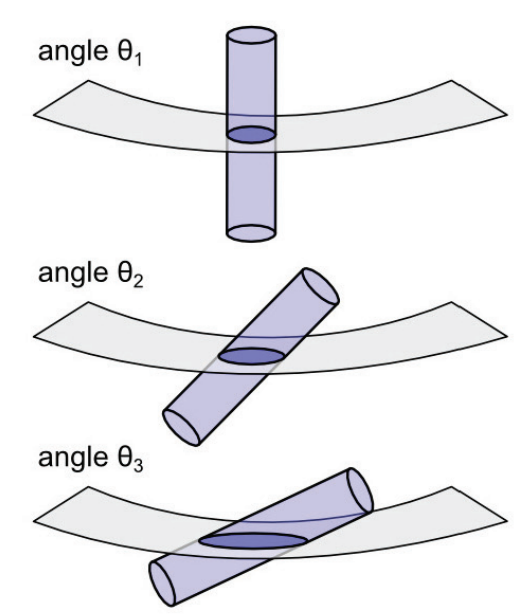


change in the prerevision and postrevision portosystemic pressure gradient $\left(\Delta P / P_{\text {avg }}\right)$. Data were also evaluated on the basis of prerevision shunt volume flow and the outcome to determine whether a flow threshold for revision could be defined. Clinical outcomes and portosystemic pressure gradient measurements were abstracted from the medical records of the recruited patients.

\section{Statistical Methods}

Linear regression analysis was performed between the volume flow and portosystemic pressure gradient metrics and plotted with a $95 \%$ confidence interval. The $\Delta Q / Q_{\text {avg }}$ volume flow metric was reported as mean and standard deviation, where the standard deviation was computed by using the standard rules of error propagation for an up to 30 -volume data set. Groups were compared by the $t$ test for independent samples and reported with 2-tailed $P$ values. Individual groups were reported as mean and standard error. All statistical analyses were performed with Prism 5 software (GraphPad, La Jolla, CA). $P<.05$ was considered a statistically significant difference.

\section{Power-Weighted Surface Integration of Doppler- Measured Velocity Vectors}

Surface integration of Doppler-measured velocity vectors is based on the Gauss divergence theorem. Volume flow, $Q$ can be defined as the total flux through a surface, $S$, that completely transects a vessel:

$$
Q=\oint_{S} \vec{v} \cdot d \vec{A}
$$

where $v$ is the Doppler-measured velocity vector, and $A$ is the surface area element. The scanning geometry illustrated in Figure 1 ensures that normality is maintained between the local velocity vector and the local surface area element; therefore, the dot product in Equation 1 simplifies to a multiplication. With this simplification and in the discrete case, mean volume flow through a c-surface can be redefined as:

$$
Q=\sum_{i \in S} v_{i} \cdot A_{i},
$$

where $v_{i}$ is the mean local Doppler-measured velocity vector for a single pixel $i$; $A_{i}$ is the local surface area of the corresponding pixel $i$; and $i$ is taken over all pixels in the c-surface. The velocity vector and surface area element must be normal (ie, perpendicular) for the application of Equation 2.

Figure 2 illustrates the principle of power-weighted surface integration of Doppler-measured velocity vectors for a single vessel with an ellipsoidal cross section in the csurface. For color flow pixels that fully intersect the vessel ( 6 dark blue pixels in Figure 2), volumetric flow through the pixel c-surface can be computed by using Equation 2 . However, a subset of color flow pixels in Figure 2 only partially intersect the vessel (14 light blue pixels in Figure 2) and thus contribute only partially to the flow estimate. The extent of partial contribution is determined by the power Doppler signal, which is assumed as directly proportional to the number of scattering red blood cells. ${ }^{33}$ A weighting factor, $w$, computed in reference to the power in pixels containing $100 \%$ blood, reflects the fraction of blood intersected by the pixel and is applied to the volume flow estimate as follows:

$$
Q=\sum_{i \in S} v_{i} \cdot\left(A_{i} \times w_{i}\right),
$$

where $0 \leq w_{i} \leq 1$, and $i$ is taken over all pixels in the c-surface. For example, $w_{i}=0.5$ indicates a pixel area that contains, or intersects, only $50 \%$ blood. Power weighting automatically identifies the vessel boundary because the weighting factor is 0 outside the vessel. Only a broad vessel outline is required to exclude extraneous flow and artifacts.

The following describes the steps for determining the weighting factor. A histogram is generated using Doppler power values corresponding to each velocity measurement

Figure 2. Power-weighted surface integration of Doppler-measured velocity vectors illustrated on a vessel with an ellipsoidal cross section (black outline) in the lateral-elevational imaging surface (c-surface). Each color flow pixel that intersects the vessel possesses a Dopplermeasured axial velocity vector that is normal for the area element. Axial velocity vectors are indicated. Color flow pixels positioned inside the vessel correspond to $100 \%$ blood; those outside the vessel correspond to $0 \%$ blood; and those partially inside the vessel correspond to values between $0 \%$ and $100 \%$ blood. Integration of flow over the c-surface yields volume flow through the shunt.
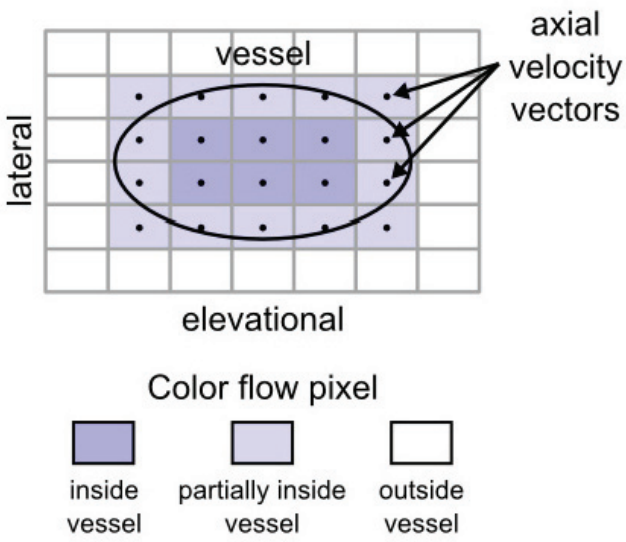
on the c-surface. The Doppler power value that corresponds to the peak in the histogram is selected automatically and defined as $p_{\max }$. A power threshold, $p_{\text {thres }}$, is chosen as $90 \%$ of $p_{\max }$ and identifies the minimum power value of those pixels positioned fully inside the vessel. This power threshold represents the minimum power value of $100 \%$ blood. Color flow pixels associated with Doppler power values greater than or equal to $p_{\text {thres }}$ are set to possess a weighting factor $w=1$. The remaining Doppler power values, all of which are greater than or equal to 0 but less than $p_{\text {thres }}$, are linearly scaled to possess a weighting factor between $0 \leq w<1$. The weighting factor is assigned irrespective of the color pixel spatial position. Evaluation of Equation 3 yields the total flow through the shunt while correcting for the partial volume effect.

\section{Results}

Figure 3 shows representative axial-lateral (Figure 3A) and elevational-lateral (Figure 3B) color flow images of a single volumetric TIPS scan for patient 4 after revision. Shunt volume flow can be assessed by the proposed method as long as the elevational-lateral surface, or constant depth c-surface, can be positioned to fully intersect the shunt in cross section, as shown in Figure 3B. This cross-sectional orientation was achieved for each recruited patient and demonstrates the broad applicability of the method.

Figure 4 shows the relationship between the $\Delta Q / Q_{\text {avg }}$ volume flow metric and the $\Delta P / P_{\text {avg }}$ portosystemic pressure gradient metric. The 12 of 20 TIPS cases for which a revision was performed are included in the linear regression of Figure 4A, which demonstrates an inverse correlation between TIPS volume flow and the portosystemic pressure gradient. An increase in shunt volume flow corresponds to a decrease in the portosystemic pressure gradient.

An updated linear regression is presented in Figure 4B, in which 2 specific revised cases were excluded from the data set. Patient $5\left(^{*}\right)$ had 2 TIPSs, of which 1 shunt was revised and the other was not revised. After revision, increased flow was observed in both shunts; therefore, the volume flow and portosystemic pressure gradient metrics could not be reliably computed. Given that the 2 shunts are components in a complex parallel vascular flow circuit, ${ }^{39}$ the flow path through a 2-TIPS structure is not clearly understood. Patient $11(+)$ had an atypical breathing pattern that was required to observe flow within the shunt lumen. Given the circumstances described, these 2 cases can be justifiably excluded, and the updated linear regression shows a stronger correlation between the volume flow and portosystemic pressure gradient metrics.
Figure 5 provides a prerevision TIPS volume flow comparison between cases not revised or revised with an unchanged portosystemic pressure gradient and cases revised with a decreased portosystemic pressure gradient. All 15 TIPS cases (12 revised and 3 not revised) are included in the comparison of Figure 5A. An updated comparison is presented in Figure 5B, in which the same 2 cases (patients 5 and 11), as described above, were excluded from the data set.

Figure 3. Color flow images of a TIPS in axial-lateral (A) and elevationallateral (c-surface; $\mathbf{B}$ ) views for patient 4 after revision. $\mathbf{A}$ and $\mathbf{B}$ coincide at the center point marked in each view. The color flow focus depth is $11.25 \mathrm{~cm}$ and is positioned at the axial center of $\mathbf{A}$. The elevational-lateral surface intersects the shunt at a depth of $11.25 \mathrm{~cm}$. B-mode image striations in $\mathbf{A}$ represent the stent mesh boundary. The color bar indicates velocity in centimeters per second.

A

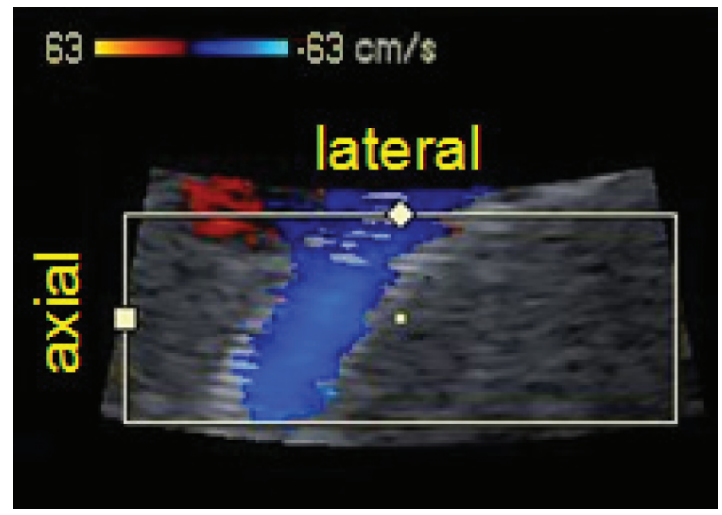

B

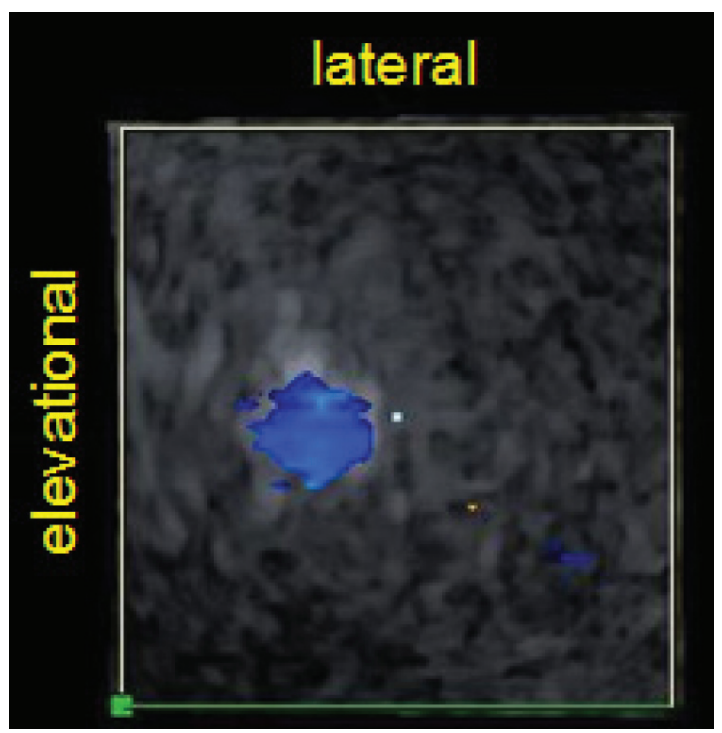


Overall, 3 data points are excluded from Figure $5 B$ because patient 5 had 2 TIPSs, and both the revised $\left({ }^{*}\right)$ and nonrevised $(+)$ prerevision flow measurements were omitted.

Comparison of prerevision flow shows a developing threshold below which a TIPS revision may be recommended. A volume flow threshold of $1534 \mathrm{~mL} / \mathrm{min}$ yields sensitivity of $66.7 \%$ and specificity of $100 \%$. A less conservative volume flow threshold of $1873 \mathrm{~mL} / \mathrm{min}$ yields sensitivity of $66.7 \%$ and specificity of $66.7 \%$. The abilities of shunt volume flow to discriminate between individuals requiring a revision and those not requiring a revision are $73 \%$ (area under the receiver operating characteristic curve $=0.73$ ) and $78 \%$ (area under the receiver operating characteristic curve $=0.78)$ for Figure 5, A and B, respectively.

\section{Discussion}

Doppler sonography has traditionally been the primary mode for TIPS evaluation and is generally accepted as an excellent method for detecting complete shunt occlusion. ${ }^{4,21,22,24-26}$

Figure 4. Relationship between the mean-normalized change in prerevision and postrevision shunt volume flow $\left(\triangle Q / Q_{\text {avg }}\right)$ and the mean-normalized change in the prerevision and postrevision portosystemic pressure gradient $\left(\triangle P / P_{\text {avg }}\right)$ for all TIPS cases for which a revision was performed $(\mathbf{A})$ and excluding 2 revised TIPS cases ( ${ }^{*}$ and + ; $\mathbf{B}$ ) for reasons described in "Results." Solid lines represent the linear regression, and dashed lines represent $95 \%$ confidence intervals. Error bars indicate standard deviation in the flow metric for an up to 30-volume data set.

A

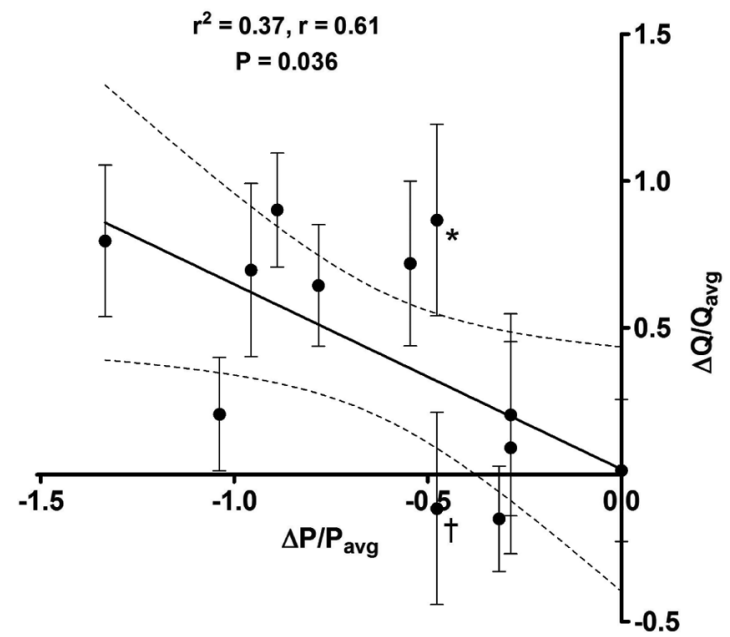

B

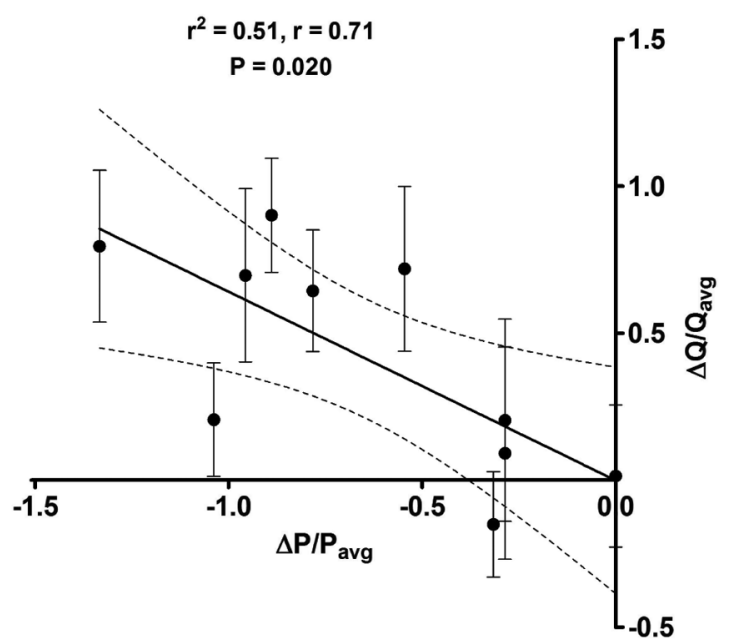

Figure 5. Prerevision TIPS volume flow comparison between cases not revised or revised with an unchanged portosystemic pressure gradient (PSPG) and cases revised with a decreased portosystemic pressure gradient for all TIPS cases (A) and excluding 2 TIPS cases (ie, 3 data points; ${ }^{*},+$, and +; B), for reasons described in "Results." Error bars indicate standard error of the mean.

A

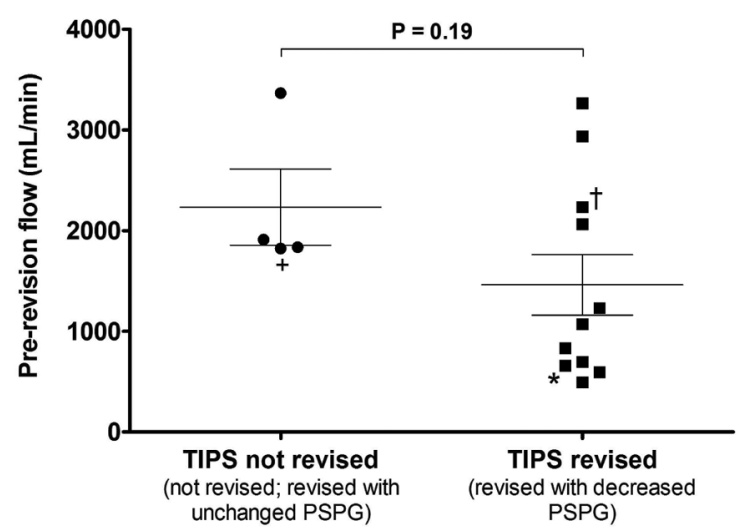

B

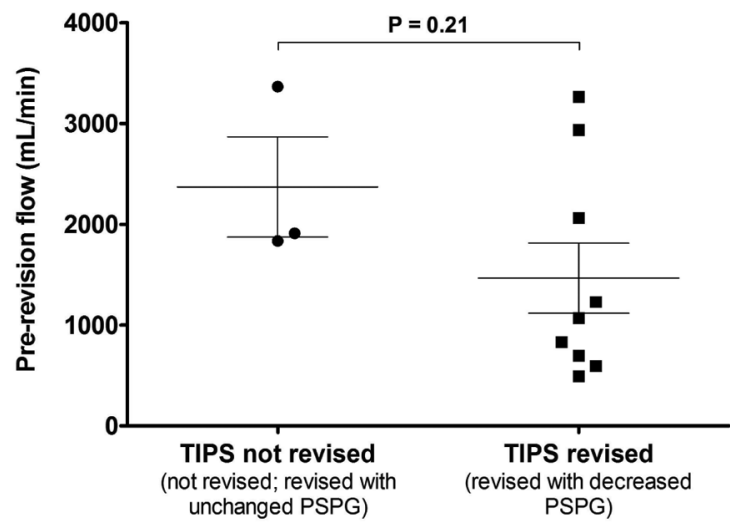


However, a completely occluded shunt is typically more difficult to revise, so a failing TIPS must be identified promptly and revised before this time. Current Doppler methods have demonstrated an opportunity for improvement.

Given the complexity in the parallel vascular flow circuit, ${ }^{39}$ shunt flow velocity depends on several factors in the overall circuit, and one can conceive of situations in which local blood flow velocities can increase, decrease, or remain unchanged in a failing TIPS. In the presence of a relative stenosis, the shunt cross-sectional area will decrease. If the TIPS behaves identically to a blood vessel that supplies an autoregulating organ, flow velocity at the stenosis would increase to maintain constant volume flow (this principle is used to estimate stenoses in carotid and renal Doppler examinations). However, a TIPS does not autoregulate and is simply intended to function as a lowresistance short circuit that diverts blood flow away from a cirrhotic liver or varices. Therefore, in a TIPS, a stenosis produces an increase in local flow resistance and may cause diversion of blood flow to other parts of the vascular flow circuit. If the decrease in blood flow equally compensates for the decrease in the cross-sectional area, the local flow velocity will remain unchanged even though a TIPS stenosis exists. In addition, the flow velocity can vary along the path of the shunt and even fluctuate with time. ${ }^{4,40}$

Variability in the flow velocity estimate is apparent in recent studies that have shown unreliable Doppler measurements when assessing TIPS function. ${ }^{27,41,42}$ In fact, updated recommended guidelines for evaluating TIPS function have reverted to recurrence of the problem for which the TIPS was originally placed as grounds for revision. ${ }^{43}$ In contrast, changes in volume flow are straightforward to interpret. For example, if TIPS flow decreases and cardiac output is presumed unchanged, blood must be shunted elsewhere such as varices, which suggests a TIPS stenosis. In this case, decreased TIPS flow would be a realistic measure of TIPS failure.

Assessment of TIPS flow and function has been demonstrated by using a 3D/4D sonographic volume flow technique that provides an alternative objective measure of shunt patency and overcomes many limitations associated with traditional pulsed wave Doppler imaging. Traditional pulsed wave Doppler imaging is unable to reliably estimate volume flow due to subjective measurement of the vessel diameter, an assumption of circular shunt geometry, subjective Doppler angle correction, and an assumption of a cylindrically symmetric flow profile. Volume flow measurements with the proposed method are independent of the vessel geometry, angle, and flow profile. Bench-top determinations of accuracy with the proposed method showed an error within 5\% achieved after acquisition of 15 volumes under pulsatile flow conditions. ${ }^{36}$ Volume flow is expected to be a more robust metric for patency than flow velocity because flow must be conserved along the path of the shunt; therefore, a measurement can be made anywhere along the TIPS.

Results of this preliminary study show an inverse correlation between the mean-normalized change in prerevision and postrevision shunt volume flow and the meannormalized change in the prerevision and postrevision portosystemic pressure gradient, which indicates that an increase in shunt volume flow corresponds to a decrease in the portosystemic pressure gradient $\left(r^{2}=0.51 ; P=.020\right)$. Therefore, a relative change in shunt blood flow appears to be an acceptable surrogate for a change in the shunt portosystemic pressure gradient for TIPS revision cases. Variability in the flow metric (error bars in Figure 4) is presumed to be due to biological variability in shunt flow.

Blood flow has an advantage over pressure in that one can perform the measurement in a standard diagnostic environment. A feasible clinical implementation would involve measurement of volume flow immediately after a successful TIPS revision: ie, an achieved portosystemic pressure gradient of less than $12 \mathrm{~mm} \mathrm{Hg}$. Screening would then involve straightforward quantitative comparison of subsequent volume flow measurements to the baseline value to assess TIPS function.

Transjugular intrahepatic portosystemic shunt volume flow measurements with the proposed 3D/4D method are in line with those presented in the literature. ${ }^{29-31}$ Comparison of prerevision shunt flow for all 15 TIPS cases shows a preliminary threshold developing at $1534 \mathrm{~mL} / \mathrm{min}$, below which a TIPS may be recommended for revision depending on complementary clinical criteria. All normal TIPS cases in this study had flow above this threshold. The group comparison in Figure 5 illustrates promise for the proposed method given published sensitivities for standard Doppler methods (eg, 35\%). ${ }^{27}$ For a representative example, consider the results from patient 9 , whose prerevision flow was measured as $1910 \pm 298.9 \mathrm{~mL} / \mathrm{min}$ (mean $\pm \mathrm{SD}$ ), which suggests that a revision may be unnecessary. On the basis of traditional criteria, this patient was referred for revision. However, the postrevision portosystemic pressure gradient was unchanged, and this outcome was reflected in postrevision flow, which was measured as $1938 \pm 355.9 \mathrm{~mL} / \mathrm{min}$. Follow-up sonography 1 month after revision showed a patent TIPS. This patient died of cardiac-related issues 5 months after revision.

An upper-bound threshold above which all TIPSs are considered normal could not be defined from the prelim- 
inary results in this study. Figure 5B shows 3 false-negative cases at the $1534-\mathrm{mL} / \mathrm{min}$ threshold defined above. Identifying such an upper-bound threshold requires further study with a larger patient population. A patient with TIPS flow above the $1534-\mathrm{mL} / \mathrm{min}$ threshold will require additional clinical criteria to confirm normal TIPS function. In contrast, TIPS flow below the threshold would by itself suggest that a TIPS revision may be necessary. However, initial measurements with the proposed volume flow method should be used as a complementary or additive clinical criterion.

Although the proposed method is independent of all traditional pulsed wave Doppler assumptions, the operator must still recognize the correct color/power gain and Doppler pulse repetition frequency to avoid aliasing. In addition, the operator must ensure that the lateral-elevational c-surface fully intersects the shunt in the cross-section. Correct selection of the color/power gain and pulse repetition frequency is necessary for any Doppler quantification and is therefore not unique to the proposed method.

This study had a few limitations. First, 2 revised cases were excluded from the linear regression analysis (Figure 4), and 2 cases (ie, 3 data points) were excluded from the prerevision volume flow comparison (Figure 5). Reasons for excluding these cases were justified due to a 2-TIPS structure and unusual contingencies in their data acquisition. Fortunately, results from these cases were outside the expected range; therefore, the resultant linear regression showed a stronger correlation $\left(r^{2}=0.51\right.$; Figure 4B). Including these cases reduces the correlation, and although the regression is less compelling, the correlation is still significant $(P=.036$; Figure $4 \mathrm{~A})$.

Second, the total acquisition time for a complete color Doppler data set consisting of $303 \mathrm{D} / 4 \mathrm{D}$ image volumes required approximately 6 to 10 minutes, with each transducer sweep requiring a breath hold. This was a technical limitation because a mechanically swept $3 \mathrm{D}$ ultrasound probe was used. However, 30 volumes were acquired because this study was interested in estimating reproducibility. In a clinical setting, an operator might be willing to make a measurement consisting of fewer acquisitions. For example, 10 acquisitions might only require 2 to 3 minutes. Furthermore, a $2 \mathrm{D}$ electronic ultrasound array can acquire 3D image volumes more rapidly than a mechanically swept $3 \mathrm{D}$ probe, and the acquisition of 30 image volumes could be completed in seconds (ie, 1 or 2 breath holds).

Third, there were limitations with the study inclusion criteria. Only patients scheduled for revision were recruited; therefore, only 3 cases with nonrevised TIPSs were enrolled in the study. Future studies intend to include addi- tional control cases to help strengthen statistical conclusions. In addition, study inclusion criteria should consider the patient's body mass index. Acoustic access to the TIPS is limited in overweight patients, which causes an increased imaging depth and reduced Doppler power at depth.

In conclusion, volume flow measurement in TIPS cases using 3D/4D Doppler sonography provides a potential alternative to standard pulsed wave Doppler metrics for evaluating shunt patency and identifying cases requiring revision. With further refinements, volume flow estimates can be incorporated as part of routine TIPS screening and evaluation. Ultimately, volume flow measurements could be applied to other vascular conditions, such as portal hypertension in the liver and liver transplants, to assess therapeutic outcomes.

\section{References}

1. Nöldge G, Rössle M, Richter GM, Perarnau JM, Palmaz JC. Modelling the transjugular intrahepatic portosystemic shunt using a metal prosthesis: requirements of the stent [in German]. Radiologe 1991; 31:102-107.

2. Brown RS Jr, Lake JR. Transjugular intrahepatic portosystemic shunt as a form of treatment for portal hypertension: indications and contraindications. Adv Intern Med 1997; 42:485-504.

3. Darcy M. Minimally invasive therapy for portal hypertension. Probl Gen Surg 1999; 16:28-43.

4. Middleton WD, Teefey SA, Darcy MD. Doppler evaluation of transjugular intrahepatic portosystemic shunts. Ultrasound Q 2003; 19:56-70.

5. Perarnau JM, Baju A, D’Alteroche L, Viguier J, Ayoub J. Feasibility and long-term evolution of TIPS in cirrhotic patients with portal thrombosis. Eur J Gastroenterol Hepatol 2010; 22:1093-1098.

6. Darcy M. Evaluation and management of transjugular intrahepatic portosystemic shunts. AJR Am J Roentgenol 2012; 199:730-736.

7. Haskal ZJ, Pentecost MJ, Soulen MC, Shlansky-Goldberg RD, Baum RA, Cope C. Transjugular intrahepatic portosystemic shunt stenosis and revision: early and midterm results. AJR Am J Roentgenol 1994; 163:439-444.

8. Lind CD, Malisch TW, Chong WK, et al. Incidence of shunt occlusion or stenosis following transjugular intrahepatic portosystemic shunt placement. Gastroenterology 1994; 106:1277-1283.

9. Kerlan RKJr, LaBerge JM, Gordon RL, Ring EJ.Transjugular intrahepatic portosystemic shunts: current status. AJR Am J Roentgenol 1995; 164: 1059-1066.

10. LaBerge JM, Somberg KA, Lake JR, et al. Two-year outcome following transjugular intrahepatic portosystemic shunt for variceal bleeding: results in 90 patients. Gastroenterology 1995; 108:1143-1151.

11. Sterling KM, Darcy MD. Stenosis of transjugular intrahepatic portosystemic shunts: presentation and management. AJR Am J Roentgenol 1997; 168:239-244.

12. Saxon RS, Ross PL, Mendel-HartvigJ, et al. Transjugular intrahepatic portosystemic shunt patency and the importance of stenosis location in the development of recurrent symptoms. Radiology 1998; 207:683-693. 
13. Zhuang ZW, Teng GJ, Jeffery RF, Gemery JM, Janne d'Othee B, Bettmann MA. Long-term results and quality of life in patients treated with transjugular intrahepatic portosystemic shunts. AJR Am J Roentgenol 2002; 179:1597-1603.

14. Bureau C, Garcia-Pagan JC, Otal P, et al. Improved clinical outcome using polytetrafluoroethylene-coated stents for TIPS: results of a randomized study. Gastroenterology 2004; 126:469-475.

15. Vignali C, Bargellini I, Grosso M, et al. TIPS with expanded polytetrafluoroethylene-covered stent: results of an Italian multicenter study.AJR Am J Roentgenol 2005; 185:472-480.

16. Hausegger KA, Karnel F, Georgieva B, et al. Transjugular intrahepatic portosystemic shunt creation with the Viatorr expanded polytetrafluoroethylene-covered stent-graft. J Vasc Interv Radiol 2004; 15:239-248.

17. Charon JP, Alaeddin FH, Pimpalwar SA, et al. Results of a retrospective multicenter trial of the Viatorr expanded polytetrafluoroethylene-covered stent-graft for transjugular intrahepatic portosystemic shunt creation. J Vasc Interv Radiol 2004; 15:1219-1230.

18. Rossi P, Salvatori FM, Fanelli F, et al. Polytetrafluoroethylene-covered nitinol stent-graft for transjugular intrahepatic portosystemic shunt creation: 3-year experience. Radiology 2004; 231:820-830.

19. Tripathi D, Ferguson J, Barkell H, et al. Improved clinical outcome with transjugular intrahepatic portosystemic stent-shunt utilizing polytetrafluoroethylene-covered stents. EurJ Gastroenterol Hepatol 2006; 18:225-232.

20. Heinzow HS, Lenz P, Köhler M, et al. Clinical outcome and predictors of survival after TIPS insertion in patients with liver cirrhosis. World J Gastroenterol 2012; 18:5211-5218.

21. Chong WK, Malisch TA, Mazer MJ, Lind CD, WorrellJA, Richards WO. Transjugular intrahepatic portosystemic shunt: US assessment with maximum flow velocity. Radiology 1993; 189:789-793.

22. Surratt RS, Middleton WD, Darcy MD, Melson GL, BrinkJA. Morphologic and hemodynamic findings at sonography before and after creation of a transjugular intrahepatic portosystemic shunt. AJR Am J Roentgenol 1993; 160:627-630.

23. Dodd GD, Zajko AB, Orons PD, Martin MS, Eichner LS, Santaguida LA. Detection of transjugular intrahepatic portosystemic shunt dysfunction: value of duplex Doppler sonography. AJR Am J Roentgenol 1995; 164: 1119-1124.

24. Foshager MC, Ferral H, Nazarian GK, Castañeda-Zuñiga WR, Letourneau JG. Duplex sonography after transjugular intrahepatic portosystemic shunts (TIPS): normal hemodynamic findings and efficacy in predicting shunt patency and stenosis. AJR Am J Roentgenol 1995; 165:1-7.

25. Feldstein VA, Patel MD, LaBerge JM. Transjugular intrahepatic portosystemic shunts: accuracy of Doppler US in determination of patency and detection of stenoses. Radiology 1996; 201:141-147.

26. Kanterman RY, Darcy MD, Middleton WD, Sterling KM, Teefey SA, Pilgram TK. Doppler sonography findings associated with transjugular intrahepatic portosystemic shunt malfunction. AJR Am J Roentgenol 1997; 168:467-472.

27. Owens CA, Bartolone C, Warner DL, et al. The inaccuracy of duplex ultrasonography in predicting patency of transjugular intrahepatic portosystemic shunts. Gastroenterology 1998; 114:975-980.
28. Murphy TP, Beecham RP, Kim HM, Webb MS, Scola F. Long-term follow-up after TIPS: use of Doppler velocity criteria for detecting elevation of the portosystemic gradient. J Vasc Interv Radiol 1998; 9:275-281.

29. Lafortune M, Martinet JP, Denys A, et al. Short- and long-term hemodynamic effects of transjugular intrahepatic portosystemic shunts: a Doppler/manometric correlative study. AJR Am J Roentgenol 1995; 164: 997-1002.

30. Lotterer E,Wengert A, FleigWE. Transjugular intrahepatic portosystemic shunt: short-term and long-term effects on hepatic and systemic hemodynamics in patients with cirrhosis. Hepatology 1999; 29:632-639.

31. Itkin M, Trerotola SO, Stavropoulos SW, et al. Portal flow and arterioportal shunting after transjugular intrahepatic portosystemic shunt creation. J Vasc Interv Radiol 2006; 17:55-62.

32. Moser U, Vieli A, Schumacher P, Pinter P, Basler S, Anliker M.ADoppler ultrasound device for assessment of blood volume flow [in German] Ultraschall Med 1992; 13:77-79.

33. Rubin JM, Adler RS, Fowlkes JB, et al. Fractional moving blood volume: estimation with power Doppler US. Radiology 1995; 197:183-190.

34. Sun Y, Ask P, Janerot-Sjöberg B, Eidenvall L, Loyd D, Wranne B. Estimation of volume flow rate by surface integration of velocity vectors from color Doppler images. J Am Soc Echocardiogr 1995; 8:904-914.

35. Kripfgans OD, Rubin JM, Hall AL, Gordon MB, Fowlkes JB. Measurement of volumetric flow. J Ultrasound Med 2006; 25:1305-1311.

36. Richards MS, Kripfgans OD, Rubin JM, Hall AL, Fowlkes JB. Mean volume flow estimation in pulsatile flow conditions. Ultrasound Med Biol 2009; 35:1880-1891.

37. Pinter SZ, Rubin JM, Kripfgans OD, et al. Three-dimensional sonographic measurement of blood volume flow in the umbilical cord. J Ultrasound Med 2012; 31:1927-1934

38. Kliewer MA, Hertzberg BS, Heneghan JP, et al. Transjugular intrahepatic portosystemic shunts (TIPS): effects of respiratory state and patient position on the measurement of Doppler velocities. AJR Am J Roentgenol 2000; 175:149-152.

39. Ho H, Sorrell K, Peng L, Yang Z, Holden A, Hunter P. Hemodynamic analysis for transjugular intrahepatic portosystemic shunt (TIPS) in the liver based on a CT-image. IEEE Trans Med Imaging 2013; 32:92-98.

40. Sheiman RG, Vrachliotis T, Brophy DP, Ransil BJ. Transmitted cardiac pulsations as an indicator of transjugular intrahepatic portosystemic shunt function: initial observations. Radiology 2002; 224:225-230.

41. Haskal ZJ, Carroll JW, Jacobs JE, et al. Sonography of transjugular intrahepatic portosystemic shunts: detection of elevated portosystemic gradients and loss of shunt function. J Vasc Interv Radiol 1997; 8:549-556.

42. Sanyal AJ, Freedman AM, Luketic VA, et al. The natural history of portal hypertension after transjugular intrahepatic portosystemic shunts. Gastroenterology 1997; 112:889-898.

43. Boyer TD,Haskal ZJ; American Association for the Study of Liver Diseases The role of transjugular intrahepatic portosystemic shunt (TIPS) in the management of portal hypertension: update 2009. Hepatology 2010; 51:306 